ISSN 0103-9954

\title{
INFLUÊNCIA DO TAMANHO DOS FRUTOS DO PALMITEIRO Euterpe edulis Mart. NA GERMINAÇÃO DAS SEMENTES E CRESCIMENTO DAS MUDAS
}

\section{INFLUENCE OF PALM Euterpe edulis Mart. FRUIT SIZE ON SEEDS GERMINATION AND SEEDLING GROWTH}

\author{
Frederico Dimas Fleig ${ }^{1}$ Sandra Marisa Rigo ${ }^{2}$
}

\begin{abstract}
RESUMO
Este trabalho foi realizado com o objetivo de avaliar o efeito do tamanho dos frutos maduros na germinação e vigor das sementes e no crescimento das mudas de Euterpe edulis Mart. Utilizou-se frutos maduros provenientes de Rio do Sul/SC. Os frutos foram peneirados em malha de 13,5 e $15 \mathrm{~mm}$, formando três amostras de diferentes tamanhos de frutos: pequenos $(\varnothing<13,5 \mathrm{~mm})$, médios ( $\varnothing$ entre 13,5 e $15 \mathrm{~mm}$ ) e grandes com diâmetros superiores a $15 \mathrm{~mm}$. Avaliou-se a germinação, do experimento conduzido em câmara de germinação, por 105 dias. Conclui-se que os frutos de tamanho médio apresentaram maiores percentagem e velocidade de germinação, seguido dos pequenos e dos grandes. No segundo experimento avaliou-se os pesos da parte aérea, das raízes e total, a altura da parte aérea, o diâmetro do coleto e o comprimento de raízes. Constatou-se que os frutos de maior tamanho originaram plantas significativamente maiores, bem como com maior peso seco quando comparadas com as originadas de frutos pequenos.
\end{abstract}

Palavras-chave: Tamanho de frutos, Euterpe edulis, germinação, vigor, crescimento.

\begin{abstract}
The objective of this research was to evaluate the effect of mature fruit size on Euterpe edulis Mart. seed germination, vigour and seedling growth. The mature fruits used in the experiment came from Rio do Sul/SC. Fruits were sifted in 13,5 and $15 \mathrm{~mm}$ mesh, forming three different fruits size samples small $(\varnothing<13,5 \mathrm{~mm})$, medium $(\varnothing$ between 13,5 e $15 \mathrm{~mm})$ and large with more than $15 \mathrm{~mm}$ diameter. The germination was evaluated in the experiment carried out at the germination chamber, for 105 days period. Medium size fruits showed a greater percentage of
\end{abstract}

1. Eng. Florestal, MSc., Prof. do Departamento de Fitotecnia. Universidade do Estado de Santa Catarina. C. Postal 281. CEP: 88520-000. Lages. SC.

2. Acadêmica do Curso de Agronomia. Bolsista PROBIC/CNPq. Universidade do Estado de Santa Catarina. C. Postal 281. CEP: 88520-000. Lages. SC. 
germination as well as a higter germination speed, followed by small and larger size fruits. The second experiment evaluated total dry weight, shot dry weight, root dry weight, shoot height, neck diameter and root length of plants derived from different fruit sizes. Larger fruits originated plants significantly taller and with a greater dry weight when compared to the small fruits.

Key words: Fruit size, Euterpe edulis, germination, vigour, growth.

\section{INTRODUÇÃO}

Atualmente, o palmiteiro encontra-se confinado as unidades de conservação estatais e, raramente, em remanescentes da mata atlântica. Por suas características ecológicas e econômicas o Euterpe edulis Mart. é uma espécie chave para o manejo sustentável das formações florestais nativas da área de domínio da floresta atlântica. Nos remanescentes destas formações florestais o palmiteiro é raro, exigindo portanto, intervenções silviculturais para o estabelecimento desta espécie. O palmiteiro pode ser estabelecido pela semeadura direta de frutos com polpa, despolpados ou pelo plantio mudas.

Para a implantação do palmital é recomendado a utilização de frutos maduros. O amadurecimento do cacho é notado pela coloração roxa (MACEDO et al., 1974).

O palmiteiro Euterpe edulis Mart. apresenta grande variação no peso de seus frutos e sementes, variando de 1000 a 2000 sementes por $\mathrm{kg}$ (EMBRAPA, 1989).

LIN (1986) observou, ao classificar um lote de frutos maduros em duas classes de diâmetro (maiores e menores que 13,5 mm), que os frutos grandes apresentavam em média um diâmetro de $15,86 \mathrm{~mm}$, com 600 frutos $/ \mathrm{kg}$, e que, nos pequenos o diâmetro era de 13,18 mm, com 1060 frutos $/ \mathrm{kg}$. MACEDO et al. (1974), comparando diferentes procedências de frutos de palmiteiro, observaram que o número de frutos por $\mathrm{kg}$ variavam de 406 a 950.

Ao comparar dois diâmetros de frutos do palmiteiro, LIN (1986) observou que os maiores apresentavam maior percentual de germinação (84\%) quando comparados aos frutos menores (65\%) e apresentavam ainda maior vigor, expresso pelo índice de velocidade de germinação (IVG). MACEDO et al. (1974) observaram que frutos grandes apresentavam percentual de germinação significativamente superiores aos de menor diâmetro (81 e $75 \%$ respectivamente para os grandes e pequenos).

Para uniformizar e aumentar o percentual de germinação dos frutos do palmiteiro, MACEDO et al. (1974) citam o despolpamento manual, a estratificação e a escarificação mecânica do tegumento.

O objetivo deste trabalho foi determinar o efeito do tamanho dos frutos do palmiteiro sobre a germinação e vigor das sementes; crescimento e peso das mudas. 


\section{MATERIAIS E MÉTODOS}

Os frutos utilizados neste estudo procederam do Município de Rio do Sul / SC. Foram colhidos de palmiteiros que vegetam nas matas ciliares do Rio Itajai-Açu, no mês de dezembro de 1994. Os frutos maduros foram passados em peneiras de crivos circulares e classificados em pequenas $(\varnothing<13,5 \mathrm{~mm})$, médias $(\varnothing$ entre 13,5 e $15 \mathrm{~mm})$ e grandes, $(\varnothing>15 \mathrm{~mm})$, constituindo-se nos tratamentos. Calculou-se as médias dos diâmetros, seus limites superiores e inferiores, e do $\mathrm{n}^{\mathrm{o}}$ de frutos $/ \mathrm{kg}$, dentro de cada um dos tratamentos.

Utilizou-se quatro amostras de 100 frutos despolpados por tratamento, em ambos os experimentos (germinação e vigor e análise de crescimento).

\section{Experimento I: Germinação e vigor}

$\mathrm{O}$ experimento foi conduzido em câmara de germinação com temperatura controlada a $27^{\circ} \mathrm{C}$ e umidade relativa do ar de $100 \%$.

Os frutos despolpados foram dispostos em bandejas plásticas, com lados de $40 \mathrm{x} 60 \mathrm{~cm} \mathrm{e}$ altura de $12 \mathrm{~cm}$, contendo areia autoclavada $\left( \pm 120{ }^{\circ} \mathrm{C}\right.$ durante 1 hora), seguido de imersão por 24 horas em hipoclorito de sódio 3\%, sendo, posteriormente, lavada em água corrente e arejada por 24 horas. A espessura da camada de areia nas bandejas foi de $10 \mathrm{~cm}$. A semeadura foi feita em linhas, com 25 frutos por linha e 12 linhas por bandeja, constituindo cada bandeja um bloco de um total de quatro blocos. Estas bandejas foram dispostas aleatoriamente em camadas, uma por prateleira, na câmara de germinação e semanalmente recebiam nova disposição. As sementes germinadas, foram contadas semanalmente, avaliando-se o número de sementes germinadas, ou seja, as que apresentam radícula, em dias após a semeadura; o total de sementes germinadas, a percentagem de germinação e o vigor. Este foi avaliado pelo índice de velocidade de germinação (IVG),

$$
\mathrm{IVG}=\Sigma\left(\mathrm{G}_{\mathbf{i}} \% / \mathrm{D}_{\mathbf{i}}\right)
$$

onde: $\mathrm{G}_{\mathrm{i}} \%=$ percentual de sementes germinadas no momento $\mathrm{i}$;

$D_{i}=n^{\circ}$ de dias entre a semeadura e a determinação de $G_{i}$.

\section{Experimento II: Análise do crescimento}

Este experimento foi conduzido em casa-de-vegetação.

Foram utilizadas bandejas plásticas iguais as descritas acima. O substrato utilizado foi a casca de arroz carbonizada, com uma camada de $10 \mathrm{~cm}$ de espessura na base e $1 \mathrm{~cm}$ do mesmo substrato na cobertura das sementes. A semeadura foi realizada em linhas, com 25 frutos por linha e 12 linhas por bandeja, constituindo cada bandeja um bloco de um total de quatro blocos. As bandejas foram dispostas aleatoriamente sobre bancada e semanalmente recebiam nova disposição, bem como, diariamente era controlada a umidade do substrato.

Após 150 dias da semeadura, foram retiradas cinco amostras de quatro plantas e realizadas as seguintes determinações: altura da parte aérea; diâmetro do coleto; comprimento das raízes; peso da 
parte aérea seca; peso da raiz seca e peso seco total.

Para a secagem da parte aérea e da raiz foi utilizado estufa a $105^{\circ} \mathrm{C}$ durante 48 horas. Para as determinações da altura das plantas e do comprimento das raízes (média das três raízes mais longas) utilizou-se régua com precisão de $\mathrm{mm}$. $\mathrm{O}$ diâmetro do coleto foi medido com paquímetro com precisão de décimos de mm. As determinações de peso foram realizadas com balança de precisão.

Todas as determinações realizadas no experimento I e II foram submetidas a análise de variância. Os resultados de porcentagem de germinação e índice de vigor foram transformados em arco-seno $\sqrt{x}$, para a análise de variância. Aplicou-se o F-teste, com a finalidade de testar diferenças significativas entre os tratamentos. Para a comparação de médias, adotou-se o teste de Tukey, a $5 \%$ de probabilidade.

\section{RESULTADOS E DISCUSSÕES}

Os valores médios dos tamanhos dos frutos encontran-se na Tabela 1. Pode-se ainda observar na mesma tabela, que o valor do desvio padrão do diâmetro médio das amostras por tratamento é reduzido, indicando haver alta homogeneidade entre as amostras de mesmo tamanho de frutos. O número de frutos por quilograma em determinada espécie é função do tamanho dos frutos e do teor de umidade dos mesmos (LIN, 1986). No caso específico do palmiteiro, ocorre variação de 406 frutos por kg para frutos grandes (MACEDO et al., 1974) e de 1060 frutos por kg no caso de frutos pequenos (LIN, 1986).

TABELA 1: Limites superior e inferior, valores médios e desvio padrão dos diâmetros e $\mathrm{n}^{\mathrm{o}}$ de frutos $/ \mathrm{kg}$ em cada classe de tamanho de fruto de Euterpe edulis.

\begin{tabular}{l|ccc}
\hline \multirow{2}{*}{ Variável } & \multicolumn{3}{c}{ Classe de tamanho dos } \\
& Pequeno & Médio & Grutos \\
\cline { 2 - 4 } & $13,40 \mathrm{~mm}$ & $14,80 \mathrm{~mm}$ & $16,00 \mathrm{~mm}$ \\
\hline Limite superior & $11,00 \mathrm{~mm}$ & $13,50 \mathrm{~mm}$ & $15,00 \mathrm{~mm}$ \\
Limite inferior & $12,30 \mathrm{~mm}$ & $14,20 \mathrm{~mm}$ & $15,60 \mathrm{~mm}$ \\
Média dos $\varnothing$ & 0,02537 & 0,00447 & 0,05129 \\
Desvio Padrão & 836,00 & 580,00 & 513,00 \\
$\mathrm{~N}^{\circ}$ frutos/kg & &
\end{tabular}

\section{Experimento I}

A germinação das sementes do palmiteiro, em função do diâmetro dos frutos, iniciou entre o $21^{\circ}$ e o $28^{\circ}$ dia do início do experimento. Os frutos de tamanho médio, aos 28 dias já apresentavam percentual de germinação de $23,5 \%$, os grandes $16 \%$ e os pequenos $0,5 \%$ (Figura 1 ).

Verifica-se que os frutos de tamanho médio apresentam maior percentual de germinação seguidos dos pequenos e dos grandes (Figura 1 e Tabela 2). Estes dados aproximam-se dos obtidos por LIN (1986) onde frutos com $\varnothing$ maiores que $13,5 \mathrm{~cm}$ apresentaram maior percentual de germinação. Este comportamento pode decorrer do fato, que os frutos de maior tamanho possuam 
sementes com índice de velocidade de germinação (IVG) menor que os de tamanho médio, e aos 105 dias apresentem um elevado percentual de sementes inalteradas por dormência ou vazias.

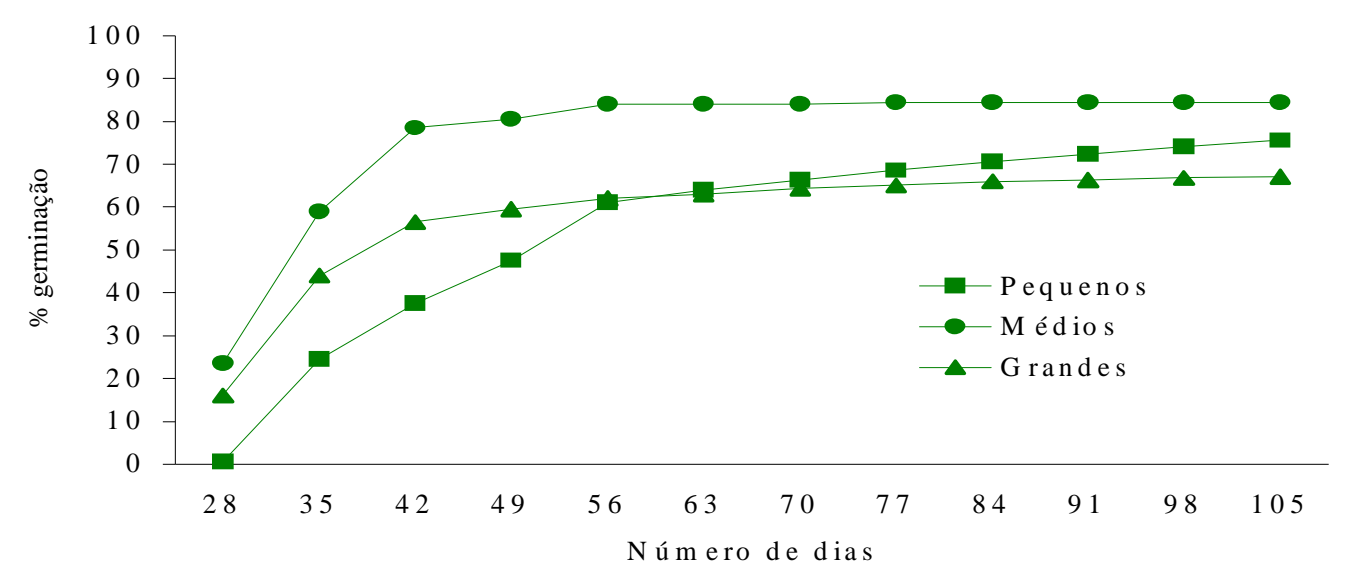

FIGURA 1: Percentual de sementes germinadas em função do tempo e do tamanho dos frutos do palmiteiro Euterpe edulis Mart.

A partir da avaliação do IVG o qual indica a velocidade de germinação, observa-se que as sementes de tamanho intermediário apresentam maior vigor germinativo em relação as demais (Tabela 2).

TABELA 2. Efeito do diâmetro do fruto de Euterpe edulis sobre a germinação e o vigor germinativo.

\begin{tabular}{l|c|c|c}
\hline \multirow{1}{*}{ Variável } & \multicolumn{3}{|c}{ Tamanho dos frutos } \\
\cline { 2 - 4 } & Pequeno & Médio & Grande \\
\hline Sem. germinadas $(\mathrm{G})$ & $75,5 \% \mathrm{~b}^{*}$ & $84,5 \% \mathrm{a}$ & $67,0 \% \mathrm{c}$ \\
sem. mortas & $3,5 \%$ & $0,0 \%$ & $0,0 \%$ \\
Sem. inalteradas & $21,0 \%$ & $15,5 \%$ & $33,0 \%$ \\
IVG** & $6,3 \mathrm{c}$ & $10,7 \mathrm{a}$ & $7,9 \mathrm{~b}$ \\
\hline
\end{tabular}

* - Médias analisadas no sentido horizontal, seguidas da mesma letra, não diferem entre si, ao nível de 5\% de probabilidade; ** IVG - índice de velocidade de germinação.

\section{Experimento II}

O tamanho dos frutos tem influência sobre o desenvolvimento das plantas como pode-se observar na Tabela 3. Os frutos maiores originaram plantas com altura, comprimento de raiz e comprimento total significativamente diferente das originadas de frutos médios e pequenos. Contudo o diâmetro do colo das plantas originadas de frutos médios ou grandes não apresentaram diferença significativa ao nível de $5 \%$ de probabilidade. 
TABELA 3: Médias da altura da parte aérea, comprimentos da raiz e total e diâmetro do colo, de frutos de Euterpe edulis.

\begin{tabular}{l|l|c|c|c}
\hline \multirow{2}{*}{$\begin{array}{c}\text { Tamanho dos } \\
\text { frutos }\end{array}$} & Altura parte & \multicolumn{2}{|c|}{ Comprimento $(\mathrm{cm})$} & \multirow{2}{*}{$\begin{array}{c}\text { Diâmetro } \\
\text { colo }(\mathrm{mm})\end{array}$} \\
\cline { 3 - 4 } & aérea $(\mathrm{cm})$ & Raiz & Total & $3,19 \mathrm{~b}$ \\
Pequeno & $7,90 \mathrm{~b} *$ & $16,50 \mathrm{~b}$ & $24,40 \mathrm{~b}$ & $3,58 \mathrm{a} \mathrm{b}$ \\
Médio & $8,20 \mathrm{~b}$ & $16,70 \mathrm{~b}$ & $24,90 \mathrm{~b}$ & $3,89 \mathrm{a}$ \\
Grande & $9,90 \mathrm{a}$ & $19,10 \mathrm{a}$ & $29,00 \mathrm{a}$ & 8,38 \\
Valor de F & 6,94 & 5,56 & 9,74 & \\
\hline
\end{tabular}

* - Médias analisadas no sentido vertical, seguidas da mesma letra, não diferem entre si, ao nível de $5 \%$ de probabilidade.

Os frutos de maior diâmetro, originam plantas com maior peso seco aéreo e total quando comparadas com as originadas de frutos médios ou pequenos (Tabela 4). Já o peso seco da raiz das mudas originadas de frutos grandes e médios são estatisticamente iguais, apesar de haver diferença estatística no comprimento das raízes destas plantas.

TABELA 4: Médias de partes secas da plântula de Euterpe edulis provenientes de frutos de diferentes tamanhos.

\begin{tabular}{l|c|c|c|c}
\hline \multirow{2}{*}{$\begin{array}{c}\text { Tamanho dos } \\
\text { frutos }\end{array}$} & \multicolumn{3}{|c|}{ Peso seco $(\mathrm{g})$} & \multirow{2}{*}{$\begin{array}{c}\text { Desvio Padrão } \\
\text { Peso seco total }\end{array}$} \\
\cline { 2 - 4 } & Aéreo & Raiz & Total & 0,041 \\
\hline Pequeno & $0,13 \mathrm{~b}^{*}$ & $0,08 \mathrm{~b}$ & $0,21 \mathrm{c}$ & 0,056 \\
Médio & $0,15 \mathrm{~b}$ & $0,11 \mathrm{a}$ & $0,26 \mathrm{~b}$ & 0,069 \\
Grande & $0,21 \mathrm{a}$ & $0,11 \mathrm{a}$ & $0,32 \mathrm{a}$ & - \\
Valor de F & 14,26 & 7,42 & 12,46 & \\
\hline
\end{tabular}

* - Médias analisadas no sentido vertical, seguidas da mesma letra, não diferem entre si, ao nível de $5 \%$ de probabilidade.

\section{CONCLUSÕES}

a) Frutos com diâmetro superior a $15 \mathrm{~mm}$ possuem percentual de germinação inferior aos de menor tamanho.

b) Frutos com diâmetro inferior a $13,5 \mathrm{~mm}$ apresentam germinação mais lenta que as de maior diâmetro. grandes.

c) Plantas de maior tamanho, bem como as de maior peso seco, são originadas de frutos

\section{AGRADECIMENTOS}

Os autores agradecem a Prof. Andréa Hansen da UDESC pela leitura e revisão dos originais, 
bem como ao CNPq e UDESC pelo auxilio financeiro.

\section{REFERÊNCIAS BIBLIOGRÁFICAS}

CANDIDO, J.F. Effect of seed size and substrate on germination of Eucalyptus citriodora Hook. Turrialba, V.20, N_2, p. 255 - 57, 1970.

COMISSÃO ESTADUAL DE PESQUISA AGRÍCOLA. Relatório anual, Florianópolis, V.2. p. 199-206, 1990.

EMPRESA BRASILEIRA DE PESQUISA AGROPECUÁRIA, CNPF. Zoneamento ecológico para plantios florestais no Estado de Santa Catarina, Versão de 21/04/89, 121 p., 1989.

LIN, S. S., Efeito do Tamanho e Maturidade sobre a Viabilidade, Germinação e Vigor do Fruto de Palmiteiro, Revista Brasileira de Sementes, Brasília, V.8, n.1, p. 57-66, 1986.

MACEDO, P.J.H, RITTERSHOFER, O.F., PESSEWFFY, A., Silvicultura e a Indústria do Palmito, Porto Alegre: Instituto de Pesquisa de Recursos Naturais Renováveis, p.61, 1974.

MENDES, C. J., CANDIDO, J. F., RESENDE, G. C. et al. Tamanho de sementes de Eucalyptus grandis (Hill) Maiden e seu efeito sobre a germinação e qualidade das mudas. IN: CONGRESSO BRASILEIRO DE SILVICULTURA, III, Manaus. Anais ... Rev. Silvicultura Ed. Especial, n. 14, p. 343-6, 1978.

REITZ, R. Palmaceas. Flora Ilustrada Catarinense, Itajai, I parte, p. 99-106, 1989. 